\title{
LIMITS ON THE FLUX OF ENERGETIC NEUTRINOS FROM THE SUN
}

\author{
J.M. LOSECCO ${ }^{a}$, J.C. VAN DER VELDE ${ }^{b}$, R.M. BIONTA ${ }^{b}$, G. BLEWITT $^{\mathrm{c}}$, C.B. BRATTON ${ }^{\mathrm{d}}$,

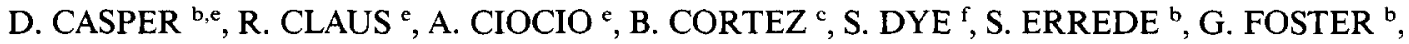 \\ W. GAJEWSKI ${ }^{\text {, }}$, K.S. GANEZER ${ }^{\text {s }}$, M. GOLDHABER ${ }^{\text {h }}$, T.J. HAINES ${ }^{\text {s }}$, T.W. JONES ${ }^{\text {i }}$, \\ D. KIELCZEWSKA ${ }^{j}$, W.R. KROPP ${ }^{g}$, J.G. LEARNED ${ }^{\text {f }}$, E. LEHMANN ${ }^{\mathrm{c}}, \mathrm{J}^{\text {J. MATTHEWS }}{ }^{\mathrm{b}}$, \\ H.S. PARK ${ }^{b}$, F, REINES ${ }^{\mathrm{g}}$, J. SCHULTZ ${ }^{\mathrm{g}}$, S. SEIDEL ${ }^{\mathrm{b}, \mathrm{e}}$, E. SHUMARD ${ }^{\mathrm{b}}$, D. SINCLAIR $^{\mathrm{b}}$, \\ H.W. SOBEL ${ }^{g}$, J.L. STONE ${ }^{\mathrm{e}}$, L. SULAK $^{\mathrm{e}}$, R. SVOBODA ${ }^{\mathrm{f}}, \mathrm{G}$. THORNTON ${ }^{\mathrm{b}}$ and C. WUEST ${ }^{\mathrm{g}}$ \\ a University of Notre Dame, Notre Dame, IN 46556, USA \\ - University of Michigan, Ann Arbor, MI 48109, USA \\ - California Institute of Technology, Pasadena, CA 91125, USA \\ d Cleveland State University, Cleveland, OH 44115, USA \\ - Boston University, Boston, MA 02215, USA \\ I University of Hawaii, Honolulu, HI 96822, USA \\ \& University of California, Irvine, CA 92717, USA \\ " Brookhaven National Laboratory, Upton, NY 11973, USA \\ ' University College, London WCIE 8BT, UK \\ Warsaw University, PL-00-681 Warsaw, Poland
}

Received 15 December 1986

\begin{abstract}
A number of authors have proposed mechanisms by which the sun could be a strong source of energetic neutrinos. We search for an excess signal of penetrating neutral particles from the direction of the sun. We employ two data samples. One sample studies energies from $400 \mathrm{MeV}$ to $2 \mathrm{GeV}$. The other studies $v_{\mu}$ interactions above $2 \mathrm{GeV}$ where the atmospheric background is lower. Our results are compared with the general background of atmospheric neutrinos from other directions. No significant excess has been found. These results can be used to set limits on possible dark matter candidates.
\end{abstract}

The nature of the dark matter implied by astronomical observations is not well understood. A number of authors [1] have pointed out that several (cold) dark matter candidates could give additional observable signals. They argue that dark matter would lose energy in passing through matter and so could become gravitationally, trapped in astronomical objects such as the sun. Such dark matter would accumulate in the sun until the point where the rate for annihilation with other particles of the same type equalled the capture rate.

The products of annihilation would include neutrinos coming from the prompt decay of heavy flavors produced in the annihilation. Neutrinos from the decay of heavy flavors tend to have energies significantly above that of neutrinos coming from typical nuclear processes in the sun. In addition both muon and electron neutrinos would be produced with comparable rates.

A number of authors [1] have calculated the flux of neutrinos at earth due to such processes in the sun. The fluxes tend to be large; comparable or greater than the ambient fluxes of neutrinos produced by cosmic ray interactions in the earth's atmosphere.

The IMB detector [2] is an 8000 metric ton deep underground detector originally designed to search for proton decay. During 417 days of livetime 401 contained interactions were observed [3] inside the 3300 metric ton fiducial volume. These events have the properties expected from neutrino interactions. The energy of these interactions extends from 200 $\mathrm{MeV}$ to about $2 \mathrm{GeV}$. The energy, position, time, direction and track multiplicity of these events is measured. In addition 187 upward going muons [4] 


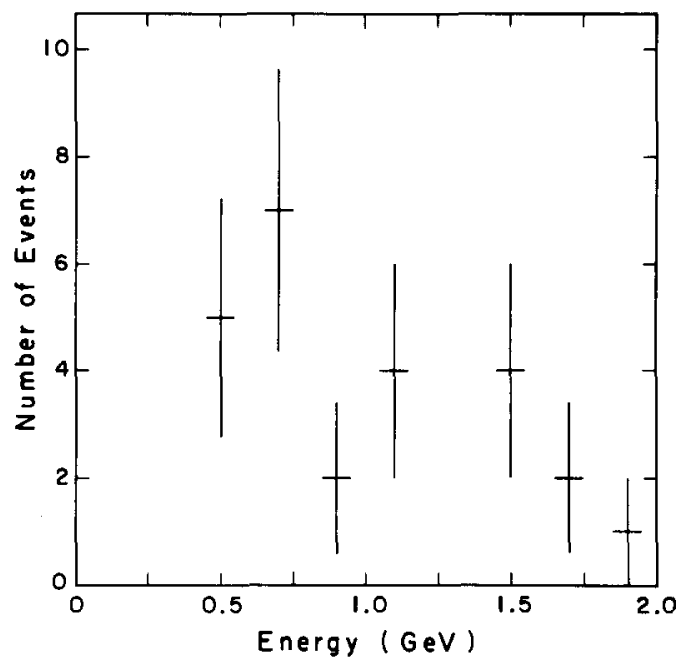

Fig. 1. Energy spectrum for all single prong contained neutrino interactions within $30^{\circ}$ of the sun.

indicative of muon neutrino interactions in the shielding rock surrounding the detector were observed in a period of 396 days of livetime. This signal comes from neutrinos with energies in excess of $2 \mathrm{GeV}$. Only the direction, time and entry point are measured for these events. Since the interaction occurs in rock outside the detector the energy cannot be measured and the observation represents an integral of all flux above the threshold. Due to the significantly higher energy of the parent neutrinos the angle between the reconstructed muon and the neutrino is small. In general it is better than the angular resolution of the detector.

The global rate observed for both of these data samples are in good agreement with expectations for atmospheric neutrinos.

A search has been conducted for an excess rate from the direction of the sun. Fig. 1 shows the neutrino energy distribution for the 25 single prong contained events found within $30^{\circ}$ of the sun. Fig. 2 shows the distribution for the total sample of 310 single prong events. The conversion from the measured visible energy to neutrino energy is an approximation [3] based on the simulated response of the detector to single prong neutrino interactions.

It is anticipated that any excess flux would be found at high energy. Table 1 lists the contents of the energy bins. The expectation is based on the solid angle subtended. A small excess is found above $1 \mathrm{GeV}$. The upper bound is computed [5] using the data sample at all other angles as a background estimate. This statistical technique [5] takes into account fluctuations in both the on source and off source measurements in deriving a $90 \%$ confidence level bound for an on source signal. The ratio of observed over expected for energies above $1 \mathrm{GeV}$ is $1.66 \pm 0.54$. This is not significant. The total sample at all energies is slightly high. The observed over expected rate is $1.20 \pm 0.25$. This may be a manifestation of the detector acceptance. The detector is longer in the east-west direction than in any other. The sun follows an east-west

Table 1

Contained events within $30^{\circ}$ of the sun.

\begin{tabular}{ccccc}
\hline $\begin{array}{l}\text { Energy } \\
\text { of events (MeV) }\end{array}$ & $\begin{array}{l}\text { Total } \\
\text { at all angles }\end{array}$ & $\begin{array}{l}\text { Events near } \\
\text { the sun }\end{array}$ & $\begin{array}{l}\text { Expected number } \\
\text { of events }\end{array}$ & $\begin{array}{l}\text { Excess events } \\
90 \% \mathrm{CL} \\
\text { upper bound }\end{array}$ \\
\hline $400-600$ & 49 & 5 & $3.3 \pm 0.5$ & 6.3 \\
$600-800$ & 111 & 7 & $7.4 \pm 0.7$ & 10.3 \\
$800-1000$ & 51 & 2 & $3.4 \pm 0.5$ & 3.3 \\
$1000-1200$ & 35 & 4 & $2.3 \pm 0.4$ & 2.3 \\
$1200-1400$ & 15 & 0 & $1.0 \pm 0.3$ & 6.9 \\
$1400-1600$ & 18 & 4 & $1.2 \pm 0.3$ & 3.7 \\
$1600-1800$ & 9 & 2 & $0.6 \pm 0.2$ & 2.3 \\
$1800-2000$ & 12 & 1 & $0.8 \pm 0.2$ & 15.1 \\
$\geqslant$ & 10 & 0 & $6.6 \pm 0.7$ & 31.2 \\
\hline
\end{tabular}




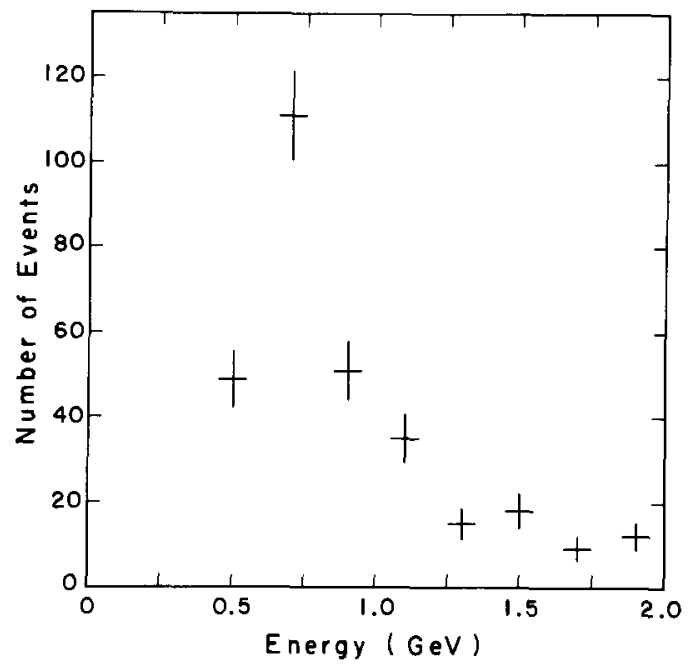

Fig. 2. Energy spectrum for all single prong contained neutrino interactions.

path and so is aligned with the detector's major axis. We can remove the detector acceptance by normalizing the high energy events to the overall flux in the suns direction. In that case we find a ratio of observed events to expected events for energies above $1 \mathrm{GeV}$ of $1.38 \pm 0.54$. So there is no evidence for excess events at high energies.

Angular cuts consistent with the resolution and the reconstructed neutrino direction can be used to reduce the overall background. The angular resolution is energy dependent. In table 2 we list the angu- lar resolution (hwhm) on a bin-by-bin basis. The angular resolution is determined by Monte Carlo studies of neutrino events taken from bubble chamber experiments. The resolution quoted includes both scattering and detector effects. Angular cuts of the quoted value have been used to get the limits in table 2. The background subtraction method of ref. [5] has been used to get the $90 \% \mathrm{CL}$ bound. It is clear that the $30^{\circ}$ angular cut chosen above is conservative for high energies $\left(E_{\mathrm{v}} \geqslant 1 \mathrm{GeV}\right)$.

The bottom line of table 2 above indicates an efficiency corrected excess of less than 4.9 events/kiloton-year in the direction of the sun. This would be the sum of $v_{\mathrm{e}}$ and $v_{\mu}$ interactions. For explicit limits we use, as an example, the calculations of Gaisser, Steigman and Tilav [1]. In the context of their calculation this result can exclude

$3 \mathrm{GeV}<m_{\mathrm{vD}_{\mathrm{D}}}<24 \mathrm{GeV}$

and

$m_{\mathrm{vD}}>80 \mathrm{GeV}, \quad m_{\mathrm{vM}}>80 \mathrm{GeV}, \quad m_{\bar{v}}>3 \mathrm{GeV}$,

and a region of

$m_{\tilde{\gamma}}<4 \mathrm{GeV}$

and

$m_{\mathrm{S}}<62 \mathrm{GeV}$.

$m_{v_{\mathrm{D}}}$ is the Dirac neutrino mass, $m_{v_{\mathrm{M}}}$ is the Majorana neutrino mass, $m_{\tilde{v}}$ is the scalar neutrino mass, $m_{\tilde{\gamma}}$ is

Table 2

Contained events near the sun.

\begin{tabular}{|c|c|c|c|c|}
\hline $\begin{array}{l}\text { Energy } \\
\text { of events }(\mathrm{MeV})\end{array}$ & $\begin{array}{l}\text { Total } \\
\text { at all angles }\end{array}$ & $\begin{array}{l}\text { Angular } \\
\text { resolution } \\
(\mathrm{hwhm})\end{array}$ & $\begin{array}{l}\text { Events near } \\
\text { the sun }\end{array}$ & $\begin{array}{l}\text { Excess events } \\
90 \% \mathrm{CL} \\
\text { upper bound }\end{array}$ \\
\hline $400-600$ & 49 & $51^{\circ}$ & 9 & 6.5 \\
\hline $600-800$ & 111 & $47^{\circ}$ & 20 & 25.5 \\
\hline $800-1000$ & 51 & $41^{\circ}$ & 6 & 5.4 \\
\hline $1000-1200$ & 35 & $36^{\circ}$ & 6 & 7.5 \\
\hline $1200-1400$ & 15 & $29^{\circ}$ & 0 & 2.3 \\
\hline $1400-1600$ & 18 & $21^{\circ}$ & 1 & 3.4 \\
\hline $1600-1800$ & 9 & $16^{\circ}$ & 0 & 2.3 \\
\hline $1800-2000$ & 12 & $16^{\circ}$ & 1 & 3.7 \\
\hline$\geqslant 2000$ & 10 & $16^{\circ}$ & 0 & 2.3 \\
\hline$\geqslant 1000$ & 99 & $20^{\circ}$ & 5 & 6.5 \\
\hline total & 310 & $18^{\circ}$ & 9 & 12.7 \\
\hline
\end{tabular}




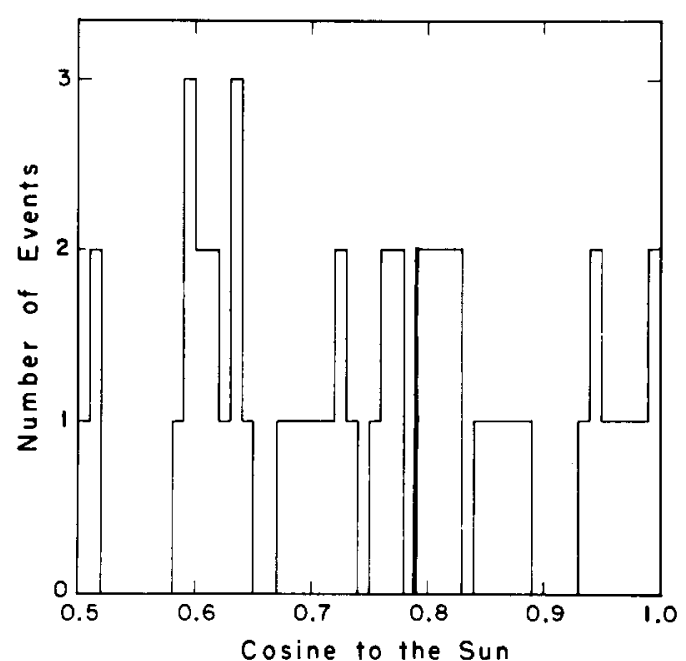

Fig. 3. Cosine of upward muons with respect to the sun. Only muons within $60^{\circ}$ of the sun are plotted.

the photino mass and $m_{\mathrm{S}}$ is the scalar quark mass. Their are many assumptions embodied in their calculation. Our results can be more restrictive as tabulated above since the actual spectrum is also bounded.

The contained sample does not include events with energies in excess of $2 \mathrm{GeV}$. These tend to exit the detector and are removed by our filtering cuts. Instead we have a sample of upward muons which come from muon neutrino interactions in the rock below the detector. Electron neutrino interactions in the rock are generally not visible to us. Only muons travelling upward are used in this sample since penetrating muons coming from the surface make it impossible to isolate the energetic neutrino sample from above. This is not a limitation in studying the sun since the detector is operated 24 hours a day and the sun spends a good deal of the time below the horizon. This data sample has good angular resolution and correlates well with the parent neutrino direction. The exact correlation [4] depends on the parent neutrino energy but in general it is less than $7^{\circ}$

Fig. 3 shows the distribution of events within $60^{\circ}$ of the sun. Table 3 shows how the distribution varies with angle and normalizes the observation to the total sample observed at all angles.

The two events found nearest the sun are more than expected but no conclusions can be drawn from such small statistics. Caution is advised in interpreting these results since the data is an integral of all neutrinos above $2 \mathrm{GeV}$ times an energy dependent detection efficiency. The efficiency tends to rise with energy due to the increase in neutrino cross section and the increase in effective detectors mass due to the high muon range. These two effects tend to offset the steep drop in the ambient neutrino flux at these energies.

The observed rate is compatible with the Volkova [6] prediction. A flux limit can be quoted from the table. Within $8^{\circ}$ of the sun we have an excess of less than 4.64 times the atmospheric neutrino rate at $90 \%$ confidence level. Very crudely [6], we estimate that the flux is $\leqslant 3.7 \times E_{\nu}^{-3.69} \mathrm{v} /\left(\mathrm{cm}^{2} \mathrm{~s} \mathrm{GeV}\right)$.

To conclude we note that both of our results are higher than expected but are consistent with expectations from atmospheric neutrinos. No result is more than 1.4 standard deviations high. More statistics would be needed to determine if the excess is significant. These limits are still useful to place constraints on possible dark matter candidates.

Table 3

Upward muon events nearest the sun.

\begin{tabular}{llllll}
\hline $\begin{array}{l}\text { Minimum } \\
\text { cosine }\end{array}$ & $\begin{array}{l}\text { Maximum } \\
\text { angle }\end{array}$ & $\begin{array}{l}\text { Number } \\
\text { of events }\end{array}$ & $\begin{array}{l}\text { Fraction } \\
\text { of total }\end{array}$ & $\begin{array}{l}\text { Fraction limit } \\
90 \% \text { CL }\end{array}$ & $\begin{array}{l}\text { Observed } \\
\text { over expected }\end{array}$ \\
\hline 0.99 & $8^{\circ}$ & 2 & $0.011 \pm 0.008$ & 0.029 & $2.14 \pm 1.52$ \\
0.98 & $11.5^{\circ}$ & 3 & $0.016 \pm 0.009$ & 0.036 & $1.60 \pm 0.90$ \\
0.97 & $14^{\circ}$ & 4 & $0.021 \pm 0.011$ & 0.043 & $1.40 \pm 0.73$ \\
0.96 & $16.25^{\circ}$ & 5 & $0.027 \pm 0.012$ & 0.050 & $1.35 \pm 0.60$ \\
0.95 & $18.2^{\circ}$ & 6 & $0.032 \pm 0.013$ & 0.056 & $1.28 \pm 0.52$ \\
0.90 & $25.8^{\circ}$ & 9 & $0.048 \pm 0.016$ & 0.076 & $0.96 \pm 0.33$ \\
0.50 & $60^{\circ}$ & 51 & $0.273 \pm 0.033$ & 0.319 & $1.09 \pm 0.13$ \\
\hline
\end{tabular}


This work was supported in part by the US Department of Energy. One of us (JML) was supported by a DOE Outstanding Junior Investigator grant. We would like to acknowledge stimulating conversations with J. Ellis, K. Olive, G. Steigman, G. Kane, T. Gaisser and J. Silk. JML would like to acknowledge the 1986 Lake Louise Winter Institute where this work was initiated.

\section{References}

[1] J. Silk, K. Olive and M. Srednicki, Phys. Rev. Lett. 55 (1985) 257 ;

B.A. Campbell, J. Ellis, K. Enqvist, D.V. Nanopoulos, J.S. Hagelin and K.A. Olive, Phys. Lett. B 173 (1986) 270;

M. Srednicki, K.A. Olive and J. Silk, High energy neutrinos from the sun and cold dark matter, UMN-TH-553/86, to be published;

J.S. Hagelin, K.W. Ng and K.A. Olive, Phys. Lett. B 180 (1986) 375;
T.K. Gaisser, G. Steigman and S. Tilav, Phys. Rev. D 34 (1986) 2206.

[2] R.M. Bionta et al., Phys. Rev. Lett. 51 (1983) 27;

S. Errede et al., Phys. Rev. Lett. 51 (1983) 245;

T.W. Jones et al., Phys. Rev. Lett. 52 (1984) 720;

B. Cortez et al., Phys. Rev. Lett. 52 (1984) 1092;

H.S. Park and G. Blewitt et al., Phys. Rev. Lett. 54 (1985) 22.

[3] J. LoSecco et al., Phys. Rev. Lett. 54 (1985) 2299;

G. Blewitt et al., Phys. Rev. Lett. 55 (1985) 2114;

T. Haines et al., Phys. Rev. Lett. 57 (1986) 1986.

[4] R. Svoboda et al., An upper limit on the flux of extraterrestrial neutrinos, UC Irvine preprint, Astrophys. J. (April 15, 1987), to be published;

R.C. Svoboda, A search for astrophysical point sources of neutrinos using a large underground water Cerenkov detector, Ph.D. Thesis, University of Hawaii at Manoa (1985), unpublished.

[5] R.F. Protheroe, Astron. Express 1 (1984) 33.

[6] L.V. Volkova, Sov. J. Nucl. Phys. 31 (1980) 784. 